\title{
Delegation Training Program for Head Nurses and its Effect on their Nurses' Competence
}

\author{
Hamdia Shawkey Abdallh ${ }^{1}$, Nermin Mohammed Eid ${ }^{2}$ and Aya Ghoneimy Hasanin ${ }^{\mathbf{3}}$ \\ (1) Master degree in Nursing administration, Ain Shams University, Egypt (2) Professor of Nursing \\ Administration, Menoufia University, Egypt and (3) Assistant Professor of Nursing administration, \\ Benha University, Egypt
}

\begin{abstract}
Background: Leaders must learn the art of effective delegation, whether a new leader or a seasoned one. Effective delegation provides several benefits to nurses, as manger are able to mobilize resources, share responsibilities. Aim: This study aimed to develop training program about delegation for head nurses and its effect on their nurses' competence Research design: A quasiexperimental design was utilized in this study. Setting: The study was conducted at Benha University Hospital. Subject: Included all available head nurses (60), and a convenient sample of staff nurses (269) who are working at the above mention setting, Tools: Four tools were used for data collection of this study; (I) Delegation Knowledge Questionnaire, (II) Attitude toward Delegation Questionnaire, (III) Performance Observational Checklist and (IV) Nurse's competence scale. Results: $91.7 \%$ and $88.3 \%$ of head nurses had good knowledge at immediate post and follow up phases respectively, $91.7 \%$ and $88.3 \%$ of head nurses had positive attitude at immediate post and follow up phases respectively, while $93.3 \%, 86.7 \%$ of them had satisfactory level of performance at immediate post and follow up phases respectively. And 91, 8\% \& 83.3\% of studied staff nurses were highly competent at immediate post and follow up program respectively. compered to preprogram. Conclusion: Training program was successful and lead to improvement in head nurses' knowledge, attitude and performance regarding delegation at immediate post follow up program phases compared to preprogram phase. In addition, there was there was a statistically significant positive correlation between head nurses' knowledge, attitude, and performance regarding delegation and their staff nurses' competences at pre, immediate post $\&$ follow up phases. Recommendations: Conducting training program and workshops periodically for head nurses about delegation to improve their managerial skills and quality of nursing care.
\end{abstract}

Key words: Delegation, Competence, Training Program, Head Nurses, Staff Nurses

\section{Introduction}

Nurses are always challenging on how they can contribute to society as professionals. They expected to take professional responsibilities for continuously providing direct care, protecting individual lives, delegation and supporting activities of daily living. To accomplish this, it is important for nurses to improve their nursing competence and utilize it in their daily practice (John, 2020).
Delegation should be a top priority for every manager it's become more and more important for leaders to grow their own supervisors, managers, and future leaders. Delegation is an essential and extremely useful management tool in nursing field; it's become increasingly important in the last years. It is one of the best known methods for efficiently managing time and leads to numerous benefits within health organization. Head nurses as the important group of health 
care workers, should learn how to manage delegation (Bittner and Gravlin, 2020).

Clearly, delegation has the potential to increase the level of productivity of heads and management of health organization. The head nurses are able to allocate more time and resources to daunting and challenging tasks that require more attention. There are three models of continuing professional development; the update model, the competence model, and the performance model. In addition to the knowledge that the Delegation is defined as the assignment of responsibilities to subordinates and conferral of authority to carry out assigned tasks, it is considered an important and effective leadership behavior. Delegation helps team members build their leadership skills, learn other aspects of work, and test out their skills and knowledge in a safe environment. When leaders have time to grow, it saves the organization recruitment and onboarding costs and contributes to a seamless transition

\section{(Abdul-Aziz, 2020).}

Competence is an ability acquired through experience and learning. It is always outcomes oriented; the goal is to evaluate performance for the effective application of knowledge and skill in the practice setting. Competence is techniques address, so the affective domains and competencies can be generic to clinical practice in any setting, specific to a clinical specialty and basic or advanced. Accordingly, competence is the set of demonstrable characteristics and skills that enable, and improve the efficiency or performance of a job (Salah, 2016).

Nursing competence is categorized into three major components: First, the ability to understand people by applying knowledge and building intrapersonal relationships. Second, the ability to provide people-centered care by providing nursing care, practicing ethically and collaborating with other professionals. Third, the ability to improve nursing quality by expanding their professional capacity and ensuring the delivery of high-quality nursing (Sayani, (2016).

The clinical teaching in the clinical practice is a specialized field which prepares head nurse to integrate their previously acquired knowledge with skills and competencies and translates theory into practice. Personnel and professional skills, attitudes and behaviors were learned and practiced in the care of patients. Nursing education program about delegation needs to play a key role in training innovative, committed and responsible nurses so that they can effectively take different roles and critical nursing duties in different situations. In this regard, one aspect of nursing practice that is bandage skill which requires preparation, good knowledge base, decision skill, and paying attention in applying delegation skills in daily tasks (Ugoani, 2020).

\section{Significance of the study}

Effective delegation creates more time for head nurse to perform the high value added tasks, creates the care plan and works of assistants to increase quality of care. Mangers in Benha University hospital have a thousand different things competing for their attention. When head nurses learn to create effective delegation, facilities the organization work and performance. In addition to, deal effectively with rapid changes and become skilled in higher-level. When head nurses learn delegation, effectively she will effect on staff nurses competence and will compromised achieved quality of patient care. (Gassas, 2017).

From the researcher observation of head nurses in Benha University hospital, she observed that there is three cases of delegation over delegation, under delegation, 
and there is no delegation. Therefore, it is important to make an educational program about delegation for head nurses and measure its effect on staff nurses competence.

\section{Aim of the study:}

The study aimed to develop training program about delegation for head nurses and its effect on their nurses' competence.

\section{Research hypothesis:}

Head nurses' knowledge, attitude and performance regarding delegation will be improved after implementing the training program and it will have a positive effect on their nurses' competence.

\section{Subjects and Method:}

\section{Research design:}

A quasi-experimental design was utilized to achieve the aim of the study.

\section{Research setting:}

The study was conducted at Benha University Hospital. In, which provides care for inpatient and outpatient services all categories of community. The hospital composed of three separated buildings namely: Medical building, Surgical building and Ophthalmology building.

\section{Subjects:}

This study subject consisted of two groups; namely head nurses group and staff nurses group.

\section{First group: Head nurses' group}

Included all the available head nurses during the time of data collection $(n=60)$

\section{Second group: Staff nurses' group}

Included a convenient sample of staff nurses who are working in the abovementioned study setting with at least three years of experience. $(n=269)$.

\section{Tools of data collection:}

Four tools were used for data collection in order to fulfill the objectives of the study:

\section{1-Delegation Knowledge Questionnaire}

It was a structured questionnaire, developed and constructed by the researcher through review of relevant literature) Ramzy (2018), Bittner, \& Gravlin, (2020); and Fenn, (2020).

It was used to assess the head nurse knowledge about delegation. It consisted of two main parts:

\section{Part 1: Personal characteristic about head} nurses as: Age, gender, level of education, marital status, years of experience and attending previous training courses about delegation.

Part II: It was concerned with the assessment of the head nurses knowledge about delegation through program phases.

\section{Scoring system:}

Each question was assigned a score of (1) for incorrect answer to (2) for correct answer. The mean and standard deviation was calculated and then converted into percentage as follows

Table (A): Scoring system of knowledge questionnaire.

\begin{tabular}{|c|c|c|}
\hline $\begin{array}{c}\text { Knowledge } \\
\text { level }\end{array}$ & score & Score percent \\
\hline good & $60-80$ & $\begin{array}{c}>75 \%(30-40 \\
\text { questions) }\end{array}$ \\
\hline Average & $48-59$ & $\begin{array}{c}60 \leq 75 \%(24-29 \\
\text { questions) }\end{array}$ \\
\hline poor & $0-47$ & $\begin{array}{c}<60 \%(0-23 \\
\text { questions) }\end{array}$ \\
\hline
\end{tabular}


2: Attitude toward delegation questionnaire

\begin{tabular}{|c|c|c|}
\hline Attitude level & score & Score percent \\
\hline $\begin{array}{c}\text { Positive } \\
\text { Attitude }\end{array}$ & $\begin{array}{c}32- \\
42\end{array}$ & $\begin{array}{c}\geq 75 \%(11-14 \\
\text { questions })\end{array}$ \\
\hline $\begin{array}{c}\text { Negative } \\
\text { Attitude }\end{array}$ & $0-31$ & $\begin{array}{c}<75 \%(0-10 \\
\text { questions })\end{array}$ \\
\hline
\end{tabular}

It was self- administered questionnaire adopted from Ramzy (2018) to assess head nurses attitude toward delegation thorough program phases. It consisted of fourteen items.

\section{Scoring system:}

Head nurses' attitude toward delegation was measured on a three points liker scale ranged from (1) disagree (2) Not sure to (3) agree. Total scores of head nurse' attitude toward delegation were summed up and converted into percent score. The mean and standard deviation was calculated and then converted into percentage as follows: Ramzy (2018).

(Table B): Scoring system of attitude questionnaire.

\section{3: Performance observational checklist}

It was adapted from Ramzy (2018) and modified by the researcher to assess head nurses' performance regarding delegation thorough program phases.it consisted of Fiftytwo items, divided into seven categories.

\section{Scoring system:}

Head nurses' performance was measured on a three points ranged from (1) not done (2) done incompletely (3) done completely. Total scores of head nurse' performance were summed up and converted into percent score as following: (Ramzy 2018).
Table (D): Scoring system of performance observational cheek list.

\begin{tabular}{|c|c|c|}
\hline $\begin{array}{c}\text { Total } \\
\text { Performance }\end{array}$ & score & Score percent \\
\hline Satisfactory & $\begin{array}{c}133- \\
156\end{array}$ & $\begin{array}{c}\geq 85 \%(45- \\
52 \text { questions })\end{array}$ \\
\hline Unsatisfactory & $0-132$ & $\begin{array}{c}<5 \%(0-44 \\
\text { questions })\end{array}$ \\
\hline
\end{tabular}

4: Nurse's competence scale: It was selfadministered questionnaire adopted from Osman, S. (2018). It was used to assess nurses 'competence thorough program phases It consists of two parts:

Part 1: Personal characteristics about staff nurses: such as: Age, gender, level of education, marital status and year of experience.

Part II: It consisted of 73 questions distributed under seven categories.

Scoring system:

Nurses 'competence was measured on a three points likert scale ranged from (0) never (1) uncertain to (2) always. Total scores of nurses 'competence were summed up and converted into percent score as following:

Table (F): Scoring system of nurse competence.

\begin{tabular}{|c|c|c||}
\hline $\begin{array}{c}\text { Nurse's } \\
\text { competence level }\end{array}$ & score & Score percent \\
\hline $\begin{array}{c}\text { Highly } \\
\text { competent }\end{array}$ & $\begin{array}{c}111- \\
146\end{array}$ & $\begin{array}{c}\geq 75 \%(56-73 \\
\text { questions) }\end{array}$ \\
\hline Competent & $\begin{array}{c}95- \\
110\end{array}$ & $\begin{array}{c}60 \leq 75 \%(48- \\
55 \text { questions })\end{array}$ \\
\hline \hline Incompetent & $0-94$ & $\begin{array}{c}<60 \%(0-47 \\
\text { questions })\end{array}$ \\
\hline
\end{tabular}


Reliability of the tools: Testing reliability of the proposed tools was done with the Cronbach's Alpha test. The results were as following:

Table (G): Reliability of study tools.

\begin{tabular}{||c|c|}
\hline Scales & $\begin{array}{c}\text { Cronbac } \\
\text { h's Alpha }\end{array}$ \\
\hline Delegation Knowledge & 0.91 \\
\hline Questionnaire & 0.92 \\
\hline $\begin{array}{c}\text { Attitude toward delegation } \\
\text { questionnaire }\end{array}$ & 0.93 \\
\hline $\begin{array}{c}\text { Performance observational } \\
\text { checklist }\end{array}$ & 0.91 \\
\hline Nurse's competence scale
\end{tabular}

Validity of the tools: Content validity was conducted to determine whether the tools cover the aim. Validity was tested by a panel of five experts, one Assistant Professor of Nursing Administration from Faculty of Nursing Banha University, two Professor of Nursing Administration from Faculty of Nursing Al Mina University, one Assistant Professor of Nursing Administration from Faculty of Nursing El-Menoufia University, one Assistant Professor of Nursing Administration from Faculty of Nursing Tanta University .The experts reviewed the tools for clarity, relevance, comprehensiveness and simplicity. Modifications were done in the light of their valuable comments and the final from was developed.

\section{Pilot study:}

A pilot study was carried out from the beginning to the end of September 2019. To ascertain the clarity and applicability of the study tools and estimate the time needed for each tool. It was done on 6 head nurses representing $10 \%$ of total study subjects these were randomly selected. The knowledge questionnaire sheet was given. The time consumed in answering the question ranged from 10-15. Then the attitude questionnaire sheet was given. The time consumed in answering the question ranged from 5-10 minutes. Then the performance observational checklist was implemented by the researcher through using observation checklist to observe their actual performance during their work in their clinical setting. The time consumed to complete this tool was ranged from 1 to1.30 hours. Based on the results no modification were done. Also nurse's competence scale was used on 27 staff nurses representing $10 \%$ of total study subjects and time from 25-30 minutes. In the light of the pilot study analysis, no modification was done and the last form was developed. Subjects was included in the study sample.

\section{Field work:}

Fieldwork was divided into four main stages, (Assessment, Preparatory, Implementation and Evaluation).

The following phases were adopted to achieve the aim of the current study.

\section{Phase (1): Program assessment}

Started from the end of September to the end of October 2019.

\section{Phase (2): Planning phase}

Started from the November to end of December 2019.

\section{Phase (3): Program implementation}

- Started from the middle of December to the end of January (2020). Each session conducted through one hour / day.

\section{Evaluation phase:}

Started from beginning of July and end of August 2020.

\section{Ethical consideration:}

Ethical approval was obtained from scientific research Ethical Committee in Faculty of Nursing at Benha University.

\section{Statistical analysis:}

All data were collected, coded, tabulated and subjected to statistical analysis. Statistical 
analysis was performed by statistical Package for Social Sciences (SPSS version 20.0), also Microsoft Office Excel is used for data handling and graphical presentation. Descriptive statistics were applied in the form of mean and standard deviation for quantitative variables, frequency and percentages for qualitative variables. Qualitative categorical variables were compared using chi-square test. Pearson correlation coefficient was calculated between variables. Whenever the expected values in one or more of the cells in $2 \times 2$ tables was less than 5, Fisher exact test was used instead. Statistical significance was considered at $\mathrm{p}$ value $\mathrm{p} \leq 0.05$, and considered highly statistically significance at $\mathrm{p}$-value $\mathrm{p} \leq 0.001$. parametrical tests (e.g., independent (t) test to compare mean scores between two sample as control and study groups, and paired(t) test to compare mean scores between the same sample at different study phases).

\section{Results}

Table (1): Showed that more than three fifth $(63.4 \%)$ of studied head nurses had age ranged between $30<40$ years. More than half $(58.3 \%)$ of studied head nurses were married. Regarding to their years of experience more than three Fifth $(63.4 \%)$ of studied head nurses had from $5<10$ years of experience. In addition, more than half $(55 \%)$ of studied head nurses had Bachelor's Degree in Nursing and more than three thirds (70\%) of them didn't attend training courses about delegation.

Table (2): Revealed that less than half $(46.1 \%)$ of staff nurses were aged from $30<$ 40 years and more than half $(59.5 \%)$ of them were married and slightly less than three quarters (73.9) of them were female nurses. Moreover, more than three fifth $(64.7 \%)$ of them were ranked between $5<10$ years of experience, and more than three fifth $(62.5 \%)$ of them had Bachelor's Degree of Nursing respectively.

Figure (1): Shows that one fifth $(20 \%)$ of studied head nurses had poor knowledge regarding delegation at preprogram phases. While it improved in increased to become 91.7 and $88.3 \%$ good knowledge at immediate post and follow up phases respectively.

Figure (2): Indicated that, more than two fifth $(45.0 \%)$ of studied head nurses had negative attitude toward delegation at preprogram phases while the majority of them had positive attitude at immediate post and follow up phases $91.7 \%, 85.0 \%$ respectively.

Figure (3): Clarifies that more than half $(56.7 \%)$ of studied head nurses had unsatisfactory level of performance regarding delegation at preprogram phases. While the majority $93.3 \%, 86.7 \%$ of them had satisfactory level of performance at immediate post and follow up phases respectively.

Figure (4): Shows that, at preprogram more than half $(51.30 \%)$ of the studied staff nurses were highly competent which improved to $91,80 \%$ \& $83.30 \%$ to immediate post and follow up program respectively.

Table (5): Demonstrates that, there were statistically significant relation between total staff nurses competence and their age at preprogram phases. In addition, there was statistical significant relation between total staff nurses' competence and their level of education at immediate post program phases.

Table (6): Revealed that, there was a statistically significant positive correlation between head nurse's knowledge, attitude, and performance regarding delegation and their staff nurse's competences at pre, immediate post $\&$ follow up phases. 


\section{Hamdia Shawkey Abdallh, Nermin Mohammed Eid and Aya Ghoneimy Hasanin}

Table (1): Distribution of studied head nurses regarding to their personal characteristics $(n=60)$

\begin{tabular}{|c|c|c|}
\hline Personal characteristics & No & $\%$ \\
\hline \multicolumn{3}{|l|}{ Age } \\
\hline $20<30$ years & 20 & 33.3 \\
\hline $30<40$ years & 38 & 63.4 \\
\hline$\geq 40$ years & 2 & 3.3 \\
\hline \multicolumn{3}{|c|}{ Mean \pm SD $\quad 31.48 \pm 3.977$} \\
\hline \multicolumn{3}{|l|}{ Marital status } \\
\hline Married & 35 & 58.3 \\
\hline Unmarried & 25 & 41.7 \\
\hline \multicolumn{3}{|l|}{\begin{tabular}{|l|} 
Years of experience \\
\end{tabular}} \\
\hline$<5$ years & 5 & 8.3 \\
\hline $5<10$ years & 38 & 63.4 \\
\hline$\geq 10$ years & 17 & 28.3 \\
\hline \multicolumn{3}{|l|}{ Educational qualification } \\
\hline Nursing diploma & 3 & 5.0 \\
\hline Associate degree of Nursing & 4 & 6.7 \\
\hline Bachelor degree of Nursing & 33 & 55.0 \\
\hline Others & 20 & 33.3 \\
\hline \multicolumn{3}{|l|}{ Attending previous training courses about delegation } \\
\hline Yes & 18 & 30.0 \\
\hline No & 42 & 70.0 \\
\hline
\end{tabular}

Table (2): Distribution of studied staff nurses regarding to their personal characteristics $(n=269)$

\begin{tabular}{|c|c|c|}
\hline Personal characteristics & No & $\%$ \\
\hline \multicolumn{3}{|l|}{ Age } \\
\hline $20<30$ years & 106 & 39.4 \\
\hline $30<40$ years & 124 & 46.1 \\
\hline$\geq 40$ years & 39 & 14.5 \\
\hline \multicolumn{3}{|l|}{ Mean \pm SD $\quad 33.16 \pm 5.853$} \\
\hline \multicolumn{3}{|l|}{ Marital status } \\
\hline Married & 160 & 59.5 \\
\hline Unmarried & 109 & 40.1 \\
\hline \multicolumn{3}{|l|}{ Gender } \\
\hline Male & 70 & 26.02 \\
\hline Female & 199 & 73.97 \\
\hline \multicolumn{3}{|l|}{ Year of experience } \\
\hline$<5$ years & 21 & 7.8 \\
\hline $5<10$ years & 174 & 64.7 \\
\hline$\geq 10$ years & 74 & 27.5 \\
\hline \multicolumn{3}{|l|}{ Education of qualification } \\
\hline Nursing diploma & 10 & 3.7 \\
\hline Associate degree of Nursing & 91 & 33.8 \\
\hline Bachelor degree of Nursing & 168 & 62.5 \\
\hline
\end{tabular}




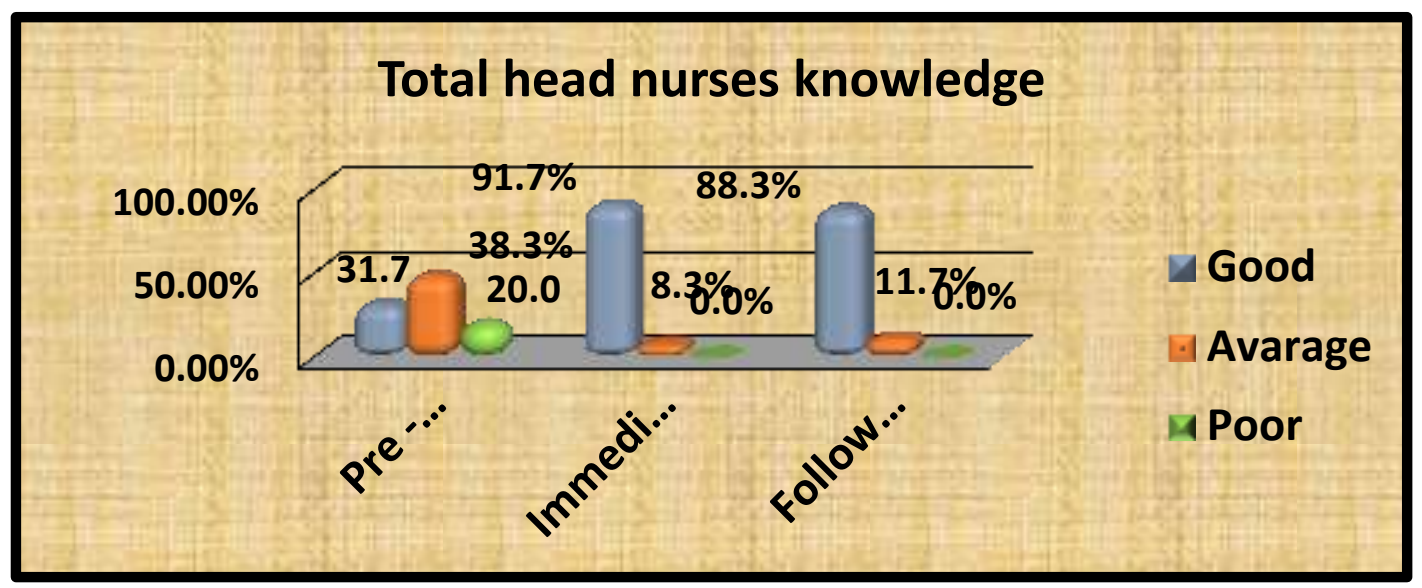

Figure (1): knowledge levels of studied head nurses regarding delegation through program phases.



Figure (2): Total levels of attitude studied head nurses toward delegation through program phases

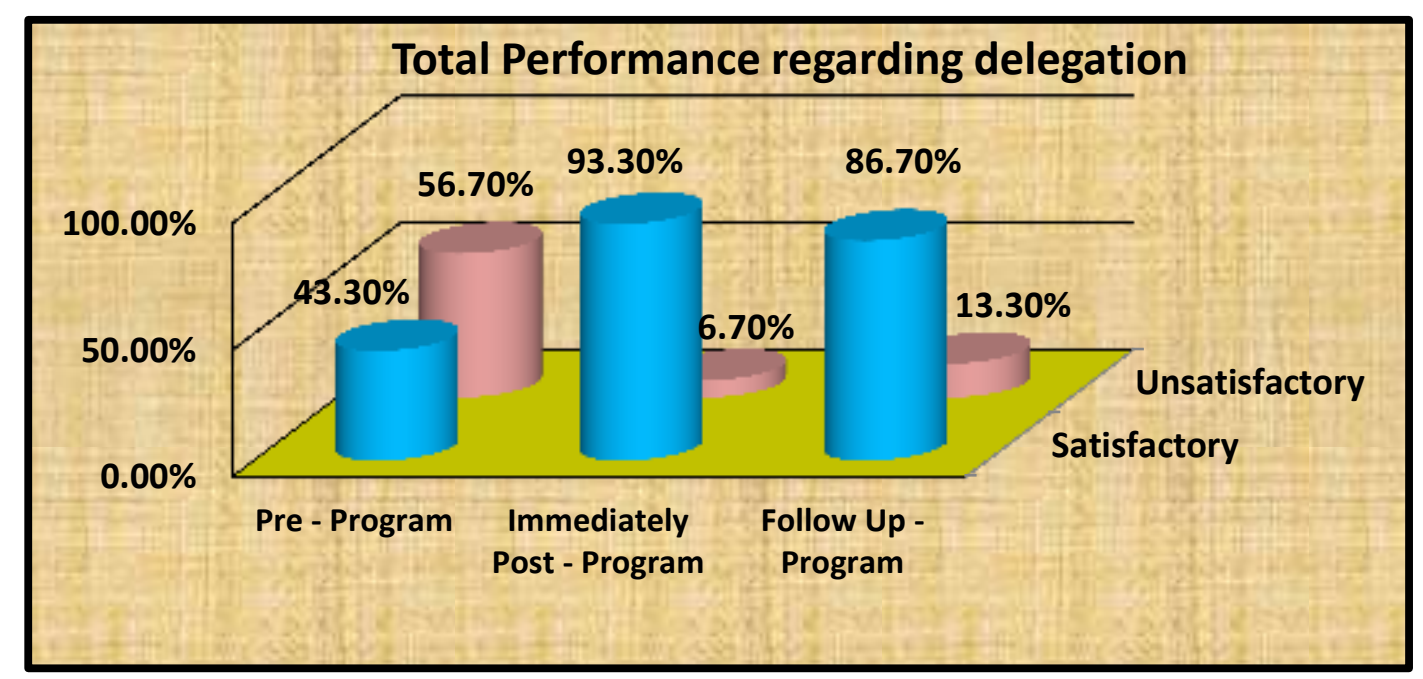

Figure (3): Distribution of total studied head nurses' performance levels regarding delegation through program phases. 
Hamdia Shawkey Abdallh, Nermin Mohammed Eid and Aya Ghoneimy Hasanin

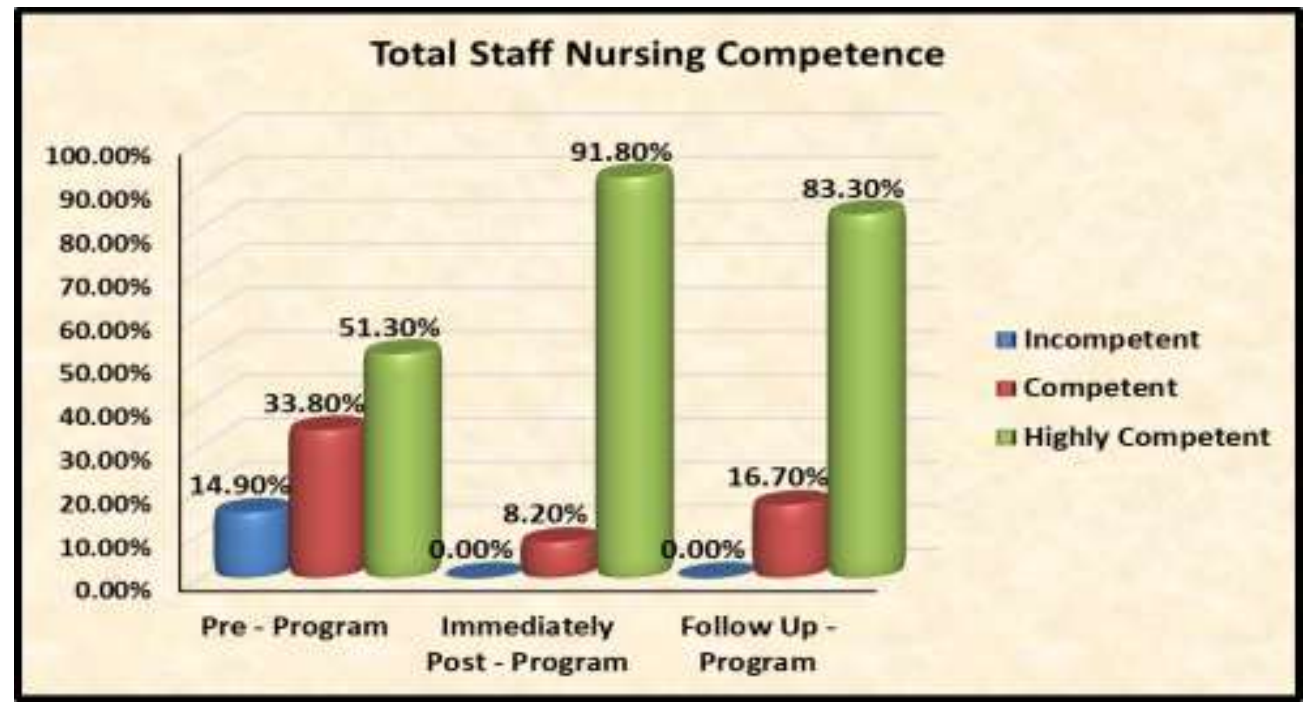

Figure (4): Distribution of total studied nurse's competence levels through program phases (n=60) 
Delegation Training Program for Head Nurses and its Effect on their Nurses' Competence

Table (4): Relation between total staff nurse's competence and their personal characteristics through program phases $(n=269)$.

\begin{tabular}{|c|c|c|c|c|c|c|c|c|c|c|c|c|c|c|c|c|c|c|c|c|}
\hline \multirow{4}{*}{$\begin{array}{c}\text { Personal } \\
\text { characteristic } \\
\text { about Staff } \\
\text { Nurses }\end{array}$} & \multicolumn{18}{|c|}{ Total Staff Nursing Competence } & \multirow{4}{*}{$\mathbf{X} 2$} & \multirow{4}{*}{ P-value } \\
\hline & \multicolumn{6}{|c|}{ Pre - program } & \multicolumn{6}{|c|}{ Immediately post - program } & \multicolumn{6}{|c|}{ Follow up - program } & & \\
\hline & \multicolumn{2}{|c|}{ Incompetence } & \multicolumn{2}{|c|}{ Competence } & \multicolumn{2}{|c|}{$\begin{array}{c}\text { High } \\
\text { Competence } \\
\end{array}$} & \multicolumn{2}{|c|}{ Incompetence } & \multicolumn{2}{|c|}{ Competence } & \multicolumn{2}{|c|}{$\begin{array}{c}\text { High } \\
\text { Competence } \\
\end{array}$} & \multicolumn{2}{|c|}{ Incompetence } & \multicolumn{2}{|c|}{ Competence } & \multicolumn{2}{|c|}{$\begin{array}{c}\text { High } \\
\text { Competence } \\
\end{array}$} & & \\
\hline & No & $\%$ & No & $\%$ & No & $\%$ & No & $\%$ & No & $\%$ & No & $\%$ & No & $\%$ & No & $\%$ & No & $\%$ & & \\
\hline \multicolumn{21}{|l|}{ Age } \\
\hline $20<30$ Years & 21 & 7.8 & 38 & 14.1 & 47 & 17.5 & 0 & 0.0 & 9 & 3.3 & 97 & 36.1 & 0 & 0.0 & 19 & 7.1 & 87 & 32.3 & \multirow{3}{*}{$\begin{array}{l}11.820 \\
0.028 \\
2.690\end{array}$} & \multirow{3}{*}{$\begin{array}{l}\text { t1:0.019* } \\
\text { t2: } 0.986 \\
\text { t3: } 0.261\end{array}$} \\
\hline $\begin{array}{c}30<40 \\
\text { Years }\end{array}$ & 17 & 6.3 & 45 & 16.7 & 62 & 23.0 & 0 & 0.0 & 10 & 3.7 & 114 & 42.4 & 0 & 0.0 & 23 & 8.6 & 101 & 37.5 & & \\
\hline $40 \leq$ Years & 2 & 0.7 & 8 & 3.0 & 29 & 10.8 & 0 & 0.0 & 3 & 1.1 & 36 & 13.4 & 0 & 0.0 & 3 & 1.1 & 36 & 13.4 & & \\
\hline \multicolumn{21}{|l|}{ Marital Status } \\
\hline Married & 27 & 10.0 & 54 & 20.1 & 79 & 29.4 & 0 & 0.0 & 15 & 5.6 & 145 & 53.9 & 0 & 0.0 & 29 & 10.8 & 131 & 48.7 & \multirow{2}{*}{$\begin{array}{l}1.354 \\
0.753 \\
0.553 \\
\end{array}$} & $\mathrm{t} 1: 0.508$ \\
\hline Unmarried & 13 & 4.8 & 37 & 13.8 & 59 & 21.9 & 0 & 0.0 & 7 & 2.6 & 102 & 37.9 & 0 & 0.0 & 16 & 5.9 & 93 & 34.6 & & $\begin{array}{l}\mathrm{t} 2: 0.386 \\
\mathrm{t} 3: 0.457\end{array}$ \\
\hline \multicolumn{21}{|c|}{ Year of Experience } \\
\hline $\begin{array}{c}\text { Less than } 5 \\
\text { years } \\
\end{array}$ & 3 & 1.1 & 7 & 2.6 & 11 & 4.1 & 0 & 0.0 & 2 & 0.7 & 19 & 7.1 & 0 & 0.0 & 2 & 0.7 & 19 & 7.1 & \multirow{3}{*}{$\begin{array}{l}0.316 \\
4.225 \\
2.238\end{array}$} & \multirow{3}{*}{$\begin{array}{l}\text { t1: } 0.989 \\
\text { t2: } 0.121 \\
\text { t3: } 0.327\end{array}$} \\
\hline $5<10$ years & 25 & 9.3 & 58 & 21.6 & 91 & 33.8 & 0 & 0.0 & 10 & 3.7 & 164 & 61.0 & 0 & 0.0 & 27 & 10.0 & 147 & 54.6 & & \\
\hline $\begin{array}{c}10 \text { years or } \\
\text { more }\end{array}$ & 12 & 4.5 & 26 & 9.7 & 36 & 13.4 & 0 & 0.0 & 10 & 3.7 & 64 & 23.8 & 0 & 0.0 & 16 & 5.9 & 58 & 21.6 & & \\
\hline \multicolumn{21}{|c|}{ Level of Education } \\
\hline $\begin{array}{l}\text { Nursing } \\
\text { Diploma }\end{array}$ & 1 & 0.4 & 3 & 1.1 & 6 & 2.2 & 0 & 0.0 & 0 & 0.0 & 10 & 3.7 & 0 & 0.0 & 0 & 0.0 & 10 & 3.7 & \multirow{3}{*}{$\begin{array}{l}4.613 \\
7.191 \\
4.221\end{array}$} & \multirow{3}{*}{$\begin{array}{c}\text { t1: } 0.329 \\
\text { t2: } \\
\text { 0.027* } \\
\text { t3: } 0.121\end{array}$} \\
\hline $\begin{array}{c}\text { Associate } \\
\text { Degree of } \\
\text { Nursing } \\
\end{array}$ & 10 & 3.7 & 27 & 10.0 & 54 & 20.1 & 0 & 0.0 & 13 & 4.8 & 78 & 29.0 & 0 & 0.0 & 20 & 7.4 & 71 & 26.4 & & \\
\hline $\begin{array}{c}\text { Bachelor } \\
\text { Degree of } \\
\text { Nursing }\end{array}$ & 29 & 10.8 & 61 & 22.7 & 78 & 29.0 & 0 & 0.0 & 9 & 3.3 & 159 & 59.1 & 0 & 0.0 & 25 & 9.3 & 143 & 53.2 & & \\
\hline
\end{tabular}


Table (5): Correlation matrix among studied head nurses knowledge, attitude, and performance regarding delegation and their staff nurse's competences through program phases.

\begin{tabular}{|c|c|c|c|c|c|c|c|c|c|c|}
\hline & \multirow{3}{*}{\multicolumn{2}{|c|}{ Studied Variables }} & \multicolumn{6}{|c|}{ Head Nurses } & \multirow{2}{*}{\multicolumn{2}{|c|}{$\begin{array}{l}\text { Staff Nursing } \\
\text { Competence }\end{array}$}} \\
\hline & & & \multicolumn{2}{|c|}{ Knowledge } & \multicolumn{2}{|c|}{ Attitude } & \multicolumn{2}{|c|}{ Performance } & & \\
\hline & & & $\mathbf{r}$ & $\mathbf{p}$ & $\mathbf{r}$ & $\mathbf{p}$ & $\mathbf{r}$ & $\bar{p}$ & $\mathbf{r}$ & $\bar{p}$ \\
\hline \multirow{9}{*}{ 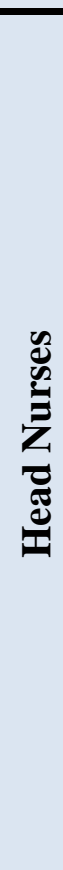 } & \multirow{3}{*}{ Knowledge } & Pre Program & $\overline{-----}$ & $\overline{-----}$ & 0.519 & $\begin{array}{c}< \\
0.001 * *\end{array}$ & 0.576 & $\begin{array}{c}< \\
0.001 * *\end{array}$ & 0.412 & $<0.05^{*}$ \\
\hline & & Post Program & ----- & 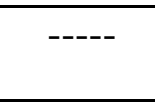 & 0.577 & $\begin{array}{c}< \\
0.001 * *\end{array}$ & 0.646 & $\begin{array}{c}<< \\
0.001 * *\end{array}$ & 0.295 & $<0.05^{*}$ \\
\hline & & $\begin{array}{l}\text { Follow Up } \\
\text { Program }\end{array}$ & $\begin{array}{l}---- \\
-1\end{array}$ & 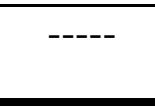 & 0.502 & $<0.05^{*}$ & 0.307 & $<0.05^{*}$ & 0.344 & $<0.05^{*}$ \\
\hline & \multirow{3}{*}{ Attitude } & Pre Program & 0.519 & $\begin{array}{c}< \\
0.001 * *\end{array}$ & ----- & ----- & 0.727 & $\begin{array}{c}< \\
0.001 * *\end{array}$ & 0.314 & $<0.05 *$ \\
\hline & & Post Program & 0.577 & $\begin{array}{c}< \\
0.001 * *\end{array}$ & ----- & ----- & 0.746 & $\begin{array}{c}<< \\
0.001 * *\end{array}$ & 0.426 & $<0.05^{*}$ \\
\hline & & $\begin{array}{l}\text { Follow Up } \\
\text { Program }\end{array}$ & 0.502 & $<0.05^{*}$ & ----- & ----- & 0.694 & $\begin{array}{c}<< \\
0.001 * *\end{array}$ & 0.334 & $<0.05 *$ \\
\hline & \multirow{3}{*}{ Performance } & Pre Program & 0.576 & $\begin{array}{c}< \\
0.001 * *\end{array}$ & 0.727 & $\begin{array}{c}< \\
0.001 * *\end{array}$ & ----. & ----- & 0.287 & $<0.05^{*}$ \\
\hline & & Post Program & 0.646 & $\begin{array}{c}< \\
0.001 * *\end{array}$ & 0.746 & $\begin{array}{c}< \\
0.001 * *\end{array}$ & & ----- & 0.367 & $<0.05 *$ \\
\hline & & $\begin{array}{l}\text { Follow Up } \\
\text { Program }\end{array}$ & 0.307 & $<0.05^{*}$ & 0.694 & $\begin{array}{c}< \\
0.001 * * \\
\end{array}$ & - & ----- & 0.341 & $<0.05^{*}$ \\
\hline \multirow{3}{*}{\multicolumn{2}{|c|}{$\begin{array}{l}\text { Staff Nursing } \\
\text { Competence }\end{array}$}} & Pre Program & 0.412 & $<0.05^{*}$ & 0.314 & $<0.05^{*}$ & 0.287 & $<0.05^{*}$ & ----- & ----- \\
\hline & & Post Program & 0.295 & $<0.05^{*}$ & 0.426 & $<0.05^{*}$ & 0.367 & $<0.05^{*}$ & $\begin{array}{ll}---- \\
\end{array}$ & ----- \\
\hline & & $\begin{array}{l}\text { Follow Up } \\
\text { Program }\end{array}$ & 0.344 & $<0.05^{*}$ & 0.334 & $<0.05^{*}$ & 0.341 & $<0.05^{*}$ & ------ & --- \\
\hline
\end{tabular}

Discussion

Today, Head Nurses and their staff are the largest group of employees in health care organizations and improving their competences has become a challenging issue in health care organizations Zakaria, (2016). Delegation has become an important nursing skill. In fact, improving delegation is a comprehensive process to improve the competence of nurses in the workplace, increase quality of life, and is essential in any organization to attract and retain its employees Abdul-Aziz et al. (2020)

Regarding total knowledge levels of delegation among head nurses, the current study indicated that, one fifth of studied head nurses had poor knowledge regarding delegation at preprogram phases while it improved to good knowledge at immediate post and slightly decreased at follow up phases. From the researcher point of view this results may be due to an effective educational program, using different teaching methods and effected booklet were head nurse's interested to acquire knowledge regarding delegation. Also, head nurses working in the studied hospital were on need to this type of knowledge about delegation and with passage of times it is expected that their knowledge decrease. Also there was a statistically difference in head nurses knowledge regarding delegation program at pre, immediate post and follow up implementation of delegation program.

This result is agreed with Hassanin, (2020) who conducted a study about " Delegation and its relation to job involvement as perceived by staff nurses in selected 
Hospital in Minia Governorate " and reported that, head nurses had good knowledge regarding delegation at immediate post and follow up phases and recommended that, workshops in service training should be carried out, followed by continuous professional development on regular basis for head nurses in the all departments. On the same line with Abd-Elmoghith, (2019) who conducted a study about "examining the time management training program on delegation skills regarding nurse manager" in Egypt and found that the highest percent of studied head nurses had good knowledge levels regarding delegation after implementation of program.

According to head nurses' attitude regarding delegation, the study finding stated that that more than two fifth of studied head nurses had negative attitude toward delegation at preprogram phases while the majority of them had positive attitude at immediate post and follow up phases respectively. Also, there was a statistically significant difference in total head nurses attitude toward delegation at pre, immediate post and follow up program phases. From the researcher point of view this improvement may be due to the success of the program which included an effective way to learn how to suitable and effective delegation by using delegation records during practical sessions and correct any mistakes for trainer head nurses and gave them feedback immediately about their attitude.

Similar finding was reported by Salem, (2021) who found that head nurses' attitude was increased significantly after training program, in his study about "attitudes of nurse managers' toward effective delegation". Also, this result was consistent with Sabah, (2018) who conduct study about "attitude and preparedness of the nurse manager's regarding effective delegation in Jinnah Hospital, who reported that in general mostly participants of the study were moderately agree toward attitude regarding delegation also, some were unsure about attitude toward delegation and they need to improve their attitude toward delegation. Moreover, Ahmed, (2017) who found in their study of "nurse manager's attitudes and preparedness towards effective delegation in a tertiary care Public Hospital Lahore" and revealed that nurse managers showed positive attitude toward delegation which improved at post training program as compared with pre training program. From the researcher point of view this results may be due to every head nurses in need to acquire theoretical and technical information that is necessary to develop their skills regarding delegation which is a part of their managerial role in their work. Also, delegation helps to complete work and enhances the role of head nurses.

Regarding to total head nurses' performance regarding delegation, the present study results demonstrated that, more than half of studied head nurses had unsatisfactory level of performance regarding delegation at preprogram phases. While the majority of them had satisfactory level of performance at immediate post and follow up phases respectively. Also, there was statistically significant differences in head nurses performance regarding delegation at pre, immediate post and follow up program phases. From the researcher point of view this might be due to the training program was successful and using different teaching methods and practical situations which improved head nurses performance regarding delegation and they acquired skills concerning how to delegation, selecting appropriate person, determine what to delegate and importance of evaluation and follow up.

This result is supported by Thomas, (2020) who conducted a study about "effect of delegation of responsibility on corporate financial performance of commercial Banks 


\section{Hamdia Shawkey Abdallh, Nermin Mohammed Eid and Aya Ghoneimy Hasanin}

in Kenya and reported that, to improve performance level, continuous professional development on regular basis for head nurses should be made in the all departments. This result is disagreed with Salem, (2021) who conducted a study about" Performance management and Operational Research: a marriage made in heaven" and reported that, there is unsatisfactory levels of performance toward delegation. This result was similar to Williamson, (2018) who investigated the impact of the delegation of authority on employee's performance at great Irbid municipality and reported that the highest percent of the studied sample had moderate level of performance related to delegation.

Regarding total staff nurses' competence through program phases, the present study indicated that there was highly statistically significance difference in total staff nurse's competence at pre, immediate post and follow up implementation of the program. From the researcher point of view, the training program about delegation improved nurses' knowledge, attitude and performance which reflected on their staff nurses and increase their competence at work.

This result was supported by Kombo, (2014) who mentioned that staff nurse's competencies and clinical function that differentiates nursing professional staff from technical to ancillary staff are mainly affected by management and supervision from their mangers. Furthermore, Abd-EIMohsen, (2019) conducted a study agreed with the present study result as it illustrated that continued nursing education programs increase both knowledge and practice and can also improve attitudes which enhance the nursese competencies as a whole.

Results of this study revealed that there was a statistically significant positive correlation between head nurses knowledge, attitude, performance and staff nurses' competence at pre, immediate post and follow up program phases. This mean that when head nurses Knowledge, attitude and performance regarding delegation increase their staff nurses' competence improved.

This finding was agreed with Bittner\& Gravlin, (2020) who reported that delegation program helps to standardize nursing management in all situation by developing and applying effective nursing management approaches through head nurses training related to more time management, communication, decision making and empowerment skills which affect their nurses' competence.

\section{Conclusion:}

Training program was successful and lead to improvement in head nurses' knowledge, attitude and performance regarding delegation at immediate post follow up program phases compared to preprogram phase. In addition, there was there was a statistically significant positive correlation between head nurses' knowledge, attitude, and performance regarding delegation and their staff nurses' competences at pre, immediate post $\&$ follow up phases. From researcher point of view this result may be due to head nurses' management and supervision is a main managerial function of head nurses and nurses are responsible for providing basic needs of patients and all their information are confirmed through the training program.

\section{Recommendations:}

Conducting training program and workshops periodically for head nurses about delegation to improve their managerial skills and quality of nursing care.

Also, conducting a study about factors affecting staff nurses' competences. 


\section{References:}

Abd-Elmoghith, N. (2019). Examining the Time Management Training Program on Delegation Skills Regarding Nurse Manager, American Journal of Nursing Research, 2019, Vol. 7, No. 4, 589-597 Available online at http://pubs sciepub.com ajnr/7/4/21 Published by Science and Education PublishingDOI:10.12691/ajnr-7-4 $-21$

Abd-EIMouhsen, S. (2019). Effect of Developing and Implementing Nursing Care Standards on outcome of Patients Undergoing Cardiac Catheterization. IOSR Journal of Nursing and Health Science (IOSR-JNHS), 54.

Abdul-Aziz, W. (2020)."The Relationship between Degree of Delegation and Head Nurses Leadership Styles at Suez Canal University Hospitals" .IOSR Journal of Nursing and Health Science (IOSR-JNHS), 9(01), 2020, pp. 45-53

Ahmed, A. (2017). Impact of management program on improving competencies of graduated nursing student during internship year; Faculty ofnursing. Menoufiya UniversityPp. 12-14

Bittner, N \& Gravlin, G. (2020). 'Critical Thinking, Delegation, and Missed Care in Nursing Practice', JONA: The Journal of Nursing Administration, vol. 39 no. 3, pp.142-146, viewed 28 March 2020, https ://journals. lww.com/jonajournal/Abstract /2009/03000/Critical_ThinkingDelegation,_a nd_Missed_Care_in.10.aspx

Fenn, (2020). Developing delegation skills American Journal of Health-System Pharmacy, Volume 77, Issue 4, 15 February 2020, Pages 254-258, https://doi.org/10.1093/ajhp/zxz313 published: 07 January 2020

Gassas, S. (2017). Nurse Managers' Attitude and Competency towards Delegation in
Jeddah City. American Journal of Nursing Science, 6 (2), P.p-73.

Hassanin, $S$ (2020). Attitude and Preparedness of the Nurse Manager's Regarding Effective Delegation in Jinnah Hospital, Lahore Lahore School of Nursing, The University of Lahore, Lahore, Pakistan M. Sabah et al. (2018) Int. J. Grad. Res. Rev.Vol-4, Issue-4: 149-155.Full text of this paper can be downloaded online at www.ijgrr.org.

John, U. (2020). Effective Delegation and Its Impact on Employee Performance (August 4, 2020). International Journal of Economics and Business Administration Vol. 6, No. 3, 2020, pp. 78-87, Available at SSRN: https://ssrn.com/abstract=3666808

Kombo, B.(2014). Effects of delegation on employee performance in savings and credit cooperative societies. In Kisii County, Kenya. The International Journal of Business and Management (7), 22-31.

Osman, S. (2018). Assessment of staff nurse's competencies related to safety measure for specific practice; Faculty of nursing. Minia University P.p 12-14

Ramzy, M. (2018). Head nurses attitude and preparedness regarding delegation and its relation to their performance;Faculty of Nursing. Benha University. Page 174-180.

Sabah, (2018): Attitude and Preparedness of the Nurse Manager's Regarding Effective Delegation in Jinnah Hospital, Lahore Lahore School of Nursing, The University of Lahore, Lahore, Pakistan M. Sabah et al. (2018) Int. J. Grad. Res. Rev.Vol-4, Issue-4: 149-155.Full text of this paper can be downloaded online at www.ijgrr.org.

Salah, M. (2016). Clinical simulation versus traditional laboratory training on nursing student's level of competence; Faculty of nursing, .Ain shams university P.p 11-13 
Salem, (2016). Nurse Manager's Attitudes and Preparedness towards Effective Delegation in Saudi Hospitals. Health Science Journal 10(3). International Journal of Innovative Research and Development 5(13).

Sayani, H., (2016). Delegation, a Strategy to Prepare Second Line Nursing Management in Health Care Setting. International journal of Innovative Research and Development, vol.5, Issue.13, Pp.149150.

Teymourzadeh, \& Shokri, A. (2019). Nurses' competency and their role in prevention and control of hospital infections: A case study in a large military teaching hospital. Advances in Human Biology, 9(2), 156- 161. https://doi.org/10.4103/aihb.aihb_1_19Web of Science ${ }^{\circledR}$ Google Scholar.

Thomas, $P$ (2020). Effect of Delegation of Responsibility on Corporate Financial Performance of Commercial Banks in Kenya: MMARAU Institutional Repositoryhttp:// hdl.handle .net /123 4567 89/10143.

Ugoani, J. (2020). Effective Delegation and Its Impact on Employee Performance. International Journal of Economics and Business Administration Vol. 6, No. 3, 2020, pp. 78-87

Williamson, E. (2018). Leaders must learn the art of effective delegation. Featured Posts, Nursing careers and jobs; Journal of advanced Nursing .January $31^{\text {st }}, 2018$.

Zakaria, A. (2016). Effectiveness of Learning Module on Time Management Ability and Delegation Skills for Head Nurses, IOSR Journal of Nursing and Health Science, 5 (2), Pp.31-40. 


\section{برنامج تلريبي عن التفويض لرئيسي التمريض وتأثيره على كفاءة ممرضيهم حمدية شوقي عبد الله - نرمين عحم عيد - أية غنيمي حسانين}

يعتبر التفويض عملية معقدة في الممارسة المهنية، ومهارة ضرورية في ممارسة التمريض؛ بسبب نقص التمريض، وارتفاع حدة المرض، بالإضافة الي التأكيد على رضا المرضى. ويعد التفويض مهارة حيوية؛ لمساعدة مشرفي التمريض على قضاء أوقاتهِج، و لأنّه من أكثر الطرق إنتاجية، ويمكن أن يوفر الوقت، ويمنحهم القدرة على أداء واجباتهم بشكل أفضل، وبناء مهار ات قيمة فيمن يفوضونه، مما يسهل تفويضهم في المستقبل. لذا هدفت هذه الدراسه الي تقييم برنامج تدريبي عن التفويض لرئيسي التمريض وتأثيره على كفاءة ممرضيهم وقد اجريت الدراسة بمسنشفي بنها الجامعي علي (·) رئيسي تمريض و عدد (Y9) مدرضة . حيث كثفت هذة الدر اسة عن وجود علاقة ارتباط ايجابيه ذات دلالة أحصائية بين مستوي معرفة وسلوك و اداء رئيسي التمريض و علاقته بكفاءة ممرضيهم في المر احل المختلفة لتفيذ البرنامج. كما اوصت الدراسة بتفيذ برنامج تدريبي عن التفويض لتحسين ممارسات الطاقم الطبي فيما يتعلق بتحسن المعرفة والسلوك والاداء مع وجود دور ات تدريبية عملية وبر امج تدريبية مكثفة لجميع مهار ات الادارة لكل فئات التمريض لتحسين كفاءتهم بأماكن در اسة مختلفة. 